\title{
Análisis de la fractalidad temporal de la precipitación en Cataluña, España (2010)
}

Óliver Meseguer-Ruiz ${ }^{1}$, oliver.meseguer@ub.edu; Javier Martín-Vide¹,jmartinvide@ub.edu

\section{RESUMEN}

Se han contabilizado los intervalos con precipitación, a resoluciones de 30 minutos hasta 48 horas, de once observatorios de Cataluña durante el año 2010. Con esto se ha analizado la fractalidad temporal de la precipitación, que tiene su expresión numérica en el concepto de la dimensión fractal (D) y que es representativa de la mayor o menor regularidad temporal de la precipitación. Asimismo, se han comparado para los mismos once observatorios las dimensiones fractales del semestre cálido y del semestre frío, con valores en general mayores en este último. El patrón espacial básico anual y del semestre cálido de D muestran un aumento de los valores desde el suroeste hacia el nordeste.

Palabras clave: Fractalidad temporal, precipitación, dimensión fractal. y Cataluña.

\section{Analysis of the temporal fractality of the precipitation in Catalonia, Spain (2010)}

\begin{abstract}
Intervals with precipitation have been counted, to orders from 30 minutes to 48 hours, in eleven observatories in Catalonia in 2010. With this we have analyzed the temporal fractality of precipitation, which has its numerical expression in the concept of fractal dimension (D), and which is representative of the degree of temporal regularity of precipitation. Also, for the same eleven observatories, the fractal dimension has been compared considering both warm and cold periods of the year, with values generally higher in the latter. The annual basic spatial pattern and the warm half of $\mathrm{D}$ shows increased values from the southwest to the northeast.
\end{abstract}

Keywords: Temporal fractality, precipitation, fractal dimension, Catalonia.

Recibido el 26 de abril de 2014, aceptado el 19 de julio de 2014.

1 Grup de Climatologia, Universitat de Barcelona. C/ Montalegre 6-8, 08001, Barcelona (España). 


\section{INTRODUCCIÓN}

La variabilidad del sistema climático en general, y de la atmósfera en particular, es notable a cualquier escala temporal que se considere. Esto es así para todas las variables climáticas, independientemente de la existencia o no de tendencias en su comportamiento cronológico. En la Península Ibérica, y por lo tanto también en Cataluña, situada al noreste de la misma, por su posición particular entre un océano y un mar casi interior, y en la zona fronteriza entre las zonas dominadas por los anticiclones subtropicales, al sur, y los vientos dominantes del oeste, al norte, la variable que muestra una mayor dispersión en sus registros es la precipitación, tanto en las cantidades como en el reparto temporal de la misma, lo que la convierte en un interesante objeto de estudio, como lo confirma el gran número de trabajos que sobre ella se han llevado a cabo en el área de estudio.

El mar Mediterráneo ocupa una extensa área de aproximadamente 2,5 millones de kilómetros cuadrados entre Europa, África y Asia, con una restringida comunicación con el océano Atlántico a través del estrecho de Gibraltar, cuya parte más angosta apenas rebasa los $14 \mathrm{~km}$. A su vez, se subdivide en dos sub-cuencas, el Mediterráneo oriental y el Mediterráneo occidental, que se comunican a través del canal de Sicilia (en ocasiones se considera una cuenca central). Por su situación geográfica, la sobreprotección que ejerce el relieve que rodea su cuenca y por apenas tener salida al océano, el mar Mediterráneo presenta comportamientos atmosféricos y marinos singulares, algo desconectados del exterior y es muy sensible a las influencias antropogénicas. El crecimiento demográfico, el cambio climático y la sobreexplotación están ejerciendo una presión excepcional en el mar y el entorno mediterráneo y en sus ecosistemas y recursos. Además, se trata de una región donde los procesos típicamente oceánicos también tienen lugar pero con unas dimensiones mucho menores, como la formación de aguas profundas, que contribuyen a mantener una célula de circulación termohalina de dimensiones iguales a las sub-cuencas, tal y como ocurre con el cinturón oceánico planetario (SCHROEDER et al. 2012). Esta realidad dota a la región mediterránea de una fuerte personalidad climática.

Fractal es una palabra inventada por MANDELBROT (1977) para reunir una clase de objetos que han desempeñado un papel histórico en el desarrollo de la Matemática del último tercio del siglo XX. Las formas y las estructuras geométricas regulares de Euclides y sus dimensiones naturales dan paso a formas nuevas, complejas, pero en las que subyace una regularidad escalar con dimensiones fraccionarias.

El concepto de fractal se utiliza para hacer referencia a objetos demasiado irregulares como para ser descritos según la geometría tradicional, pero que tienen la importante propiedad de ser invariantes por cambio de escala. La geometría fractal (MANDELBROT 1977) es una extensión de la geometría clásica y engloba la descripción, la clasificación y el análisis de subespacios geométricamente "complicados". Generalmente, la estructura y organización de un conjunto fractal no hace posible especificar dónde -en lenguaje llano- se sitúa cada punto que lo compone. Por ello debe definirse alguna relación entre las diversas estructuras observadas en el mismo para varios niveles de resolución. Esta relación se formula cuantitativamente a través del concepto de dimensión fractal, que describe el comportamiento escalar de las estructuras fractales.

Al igual que sucede con los objetos fractales, los procesos y sistemas invariantes por cambio de escala, no poseen una escala que los caracterice. Teniendo esto en cuenta, un proceso fractal es aquel en el que el mismo proceso elemental tiene lugar a distintas escalas, es decir, en el que una 
parte reproduce el todo. Para el intervalo de escalas analizadas, los estadísticos del proceso siguen leyes potenciales caracterizadas por sus exponentes. La relación entre los estadísticos para las diferentes escalas consistirá simplemente en cocientes de escala.

A modo de generalización, las dimensiones fractales, al contrario que las dimensiones corrientes o euclídeas que son siempre números enteros no negativos (0 para el caso de un punto, 1 para una recta, 2 para un plano, 3 para un espacio de tres dimensiones, etc.), pueden presentar un valor real no negativo. Para obtener una imagen más clara de esto, supongamos un punto que se desplaza sobre un plano describiendo un movimiento browniano, es decir, un movimiento aleatorio que tienda a completar o rellenar paulatinamente dicho plano. En un principio, su dimensión será 0 , pero en el mismo instante en el que empiece a describir dicho movimiento, en su primer trayecto recto, su dimensión fractal pasará a ser 1, e inmediatamente después empezará a aumentar los decimales, pero sin llegar a dos, ya que no completará dicho plano.

Tradicionalmente, el concepto de fractalidad ha sido utilizado en objetos con dimensiones espaciales, donde la noción de dimensión fractal es más evidente y de más fácil comprensión. Así, se han encontrado patrones espaciales de distribución de la precipitación que se corresponden con un objeto fractal (PÉREZ et al. 2009). Sin embargo, mucho más raro es el concepto de fractalidad temporal. En este caso, se suele considerar los intervalos temporales en los que hay lluvia o no la hay, independientemente de la cantidad de lluvia registrada, a partir de los cuales se realiza una aproximación fractal.

El análisis de la fractalidad temporal de la precipitación se ha usado para el estudio de las dinámicas climáticas que han afectado al planeta. Así, en algunos estudios se ha comparado la dimensión fractal de las curvas que representan los cambios en el nivel del mar con una dimensión fractal moderna a partir de registros anuales de precipitación, obteniendo que los cambios de nivel del mar durante los últimos 150.000 a 250.000 años presentan unas dimensiones fractales comparables a las obtenidas para la precipitación. Sin embargo, para períodos anteriores, los valores de la dimensión fractal calculados son bastante diferentes a aquellos que se deducen de los cambios de nivel del mar, por lo que cabría deducir que estos cambios estarían menos relacionados con la variabilidad climática y más con la tectónica de placas (HSUI et al 1993).

Efectivamente, este tipo de dinámicas se han identificado en estudios en la España peninsular a partir de series largas (noventa años) de precipitación acumulada anual, y su análisis revela que la distribución de esta variable se ajusta a una distribución fractal (OÑATE RUBALCABA 1997). Los valores obtenidos, con una media de 1,32 de dimensión fractal para todo el territorio, son del mismo orden de magnitud que las dimensiones fractales obtenidas a partir de otros registros macrometeorológicos y paleoclimáticos. La comparación de ambas escalas temporales sugiere que estos valores son característicos de un teórico cambio climático en todo el rango del mes de junio de 10 a 1.000 .000 de años. Estos resultados contribuyen a la creación de una hipótesis válida para la extrapolación de cambios climáticos de una escala a otra y también en el diseño de modelos de aplicación en Hidrología.

El cálculo de la dimensión fractal a nivel anual también puede servir para identificar tendencias, que después han de ser confirmadas con los tests de tendencias al uso (como el test de Mann Kendall), con el fin, en algunos casos, de determinar si en el futuro, según los diferentes escenarios de cambio climático, las cantidades acumuladas serán mayores o menores que las actuales. Tal es el caso estudiado en la provincia de La Pampa (Argentina), donde 
se ha confirmado que las proyecciones realizadas por los modelos en el IPCC para esta región se ajustan a la realidad de los datos observados (PÉREZ et al. 2009). Estudios similares en Venezuela (AMARO et al. 2004), sobre diez observatorios en un período de 80 años (1921-2000), muestran que las series de precipitación se ajustan a una distribución fractal, y que la mayoría de las series tienen persistencia a largo plazo.

La dimensión fractal constituye un índice que puede ser explicativo del comportamiento temporal de la precipitación a resolución fina, lo que tiene un gran interés.

\section{MATERIALES Y MÉTODOS}

El principal objetivo del trabajo es conocer la dimensión fractal temporal de la precipitación en los diferentes observatorios estudiados, tanto a resolución anual, como para el semestre frío (de octubre a marzo) y el cálido (de abril a septiembre) del año 2010. Como objetivos específicos están hallar las pautas comportamentales de las diferencias de las dimensiones fractales anual y de los semestres frío y cálido, y obtener el patrón espacial que explique los diferentes valores de fractalidad temporal de los observatorios.

El presente trabajo se ha llevado a cabo a partir de los datos que se encuentran en el sitio web del Servei Meteorològic de Catalunya (www.meteo.cat). Desde este sitio han sido descargados los datos proporcionados por la Xarxa d'Estacions Meteorològiques Automátiques (XEMA) de una selección de observatorios con registros completos del año 2010, y a una resolución semihoraria (cada 30 minutos).

El área de estudio en este trabajo se corresponde con Cataluña, al noreste de la Península Ibérica, y con una superficie aproximada de $32.000 \mathrm{~km}^{2}$, a través de once observatorios meteorológicos, repartidos regularmente en el territorio, con registros completos a resolución semihoraria en el año 2010 (Fig. 1). La variedad geográfica del área de estudio queda cubierta, en este primer análisis, con cinco observatorios litorales o prelitorales, cuatro en la depresión central catalana o en sus proximidades, y dos en la zona pirenaica.

En primer lugar, se han descargado todos los registros pluviométricos a resolución semihoraria para la totalidad de los días del 2010, y para los once observatorios analizados. Se considera el período de 30 minutos como el intervalo unitario de base para llevar a cabo el análisis. A continuación se han establecido períodos que contuvieran $1,2,4,6,8,12,16,24,48 \mathrm{y}$ 96 intervalos unitarios, es decir, períodos de 30 minutos, 1, 2, 3, 4, 6, 8, 12, 24 y 48 horas respectivamente, $\mathrm{y}$ se ha contabilizado en cuántos de ellos se registró alguna cantidad de precipitación.

Con estos datos, para determinar la dimensión fractal de la distribución temporal de la precipitación, el método más sencillo consiste en estimar la pendiente de la recta de regresión resultante de representar los pares de valores obtenidos a partir de los logaritmos en base 10 de n, extensión o longitud del intervalo, y de $1(n)$, número de intervalos con precipitación. En efecto, los logaritmos de esos pares de valores para cada observatorio se alinean con notable aproximación. La dimensión fractal $\mathrm{D}$ viene dada por $1+\alpha$, donde $\alpha$ es el valor absoluto de la pendiente de la recta de regresión.

\section{RESULTADOS}

\section{Determinación de las dimensiones fractales}

A continuación se presentan los valores de los conteos de intervalos con precipitación para cada uno de los observatorios durante todo el año 2010 y durante los semestres cálido (de abril a septiembre) y frío (de 
enero a marzo y de octubre a diciembre) del mismo (Tabla 1).

A partir de estos valores se representan las rectas de regresión de los pares de logaritmos citados, que, a modo de ilustración, para el caso de Anglès queda tal y como se aprecia en la figura 2 (año completo en negro, semestre cálido en rojo y semestre frío en azul).

De este modo se han obtenido los diferentes valores de las dimensiones fractales (D) para los once observatorios seleccionados, así como, previamente, los coeficientes de determinación, o la $\mathrm{r}$ de Pearson al cuadrado, que muestran una buena correlación de las dos series de logaritmos en todos los casos (Tabla 2).

Es de especial interés conocer cuál es la diferencia entre los dos semestres, ya que los mecanismos generadores de las precipitaciones son muy distintos (Tabla 2).
En términos generales, los valores anuales de las dimensiones fractales varían entre 1,4479 en Illa de Buda y 1,5504 de Fabra. Para el semestre cálido, el valor mínimo, 1,4052 , vuelve a registrarse en Illa de Buda, mientras que el máximo se registra en Anglès, 1,5554. En el semestre frío, el valor mínimo se recoge en el mismo emplazamiento que en los casos anteriores, con un valor de 1,4735 , mientras que el valor máximo se da de nuevo en Barcelona (Fabra) $(1,6066)$. La mayor diferencia entre ambos semestres $(-0,1331)$ se localiza en Fabra, mientras que la diferencia menos acusada corresponde a Banyoles (-0,0156), pero con un valor muy similar al de Das, aunque éste es positivo $(0,0158)$. Hay un predominio de diferencias negativas (9 de 11 observatorios) o, lo que es lo mismo, el valor de $\mathrm{D}$ del semestre frío es claramente mayor que el del cálido.

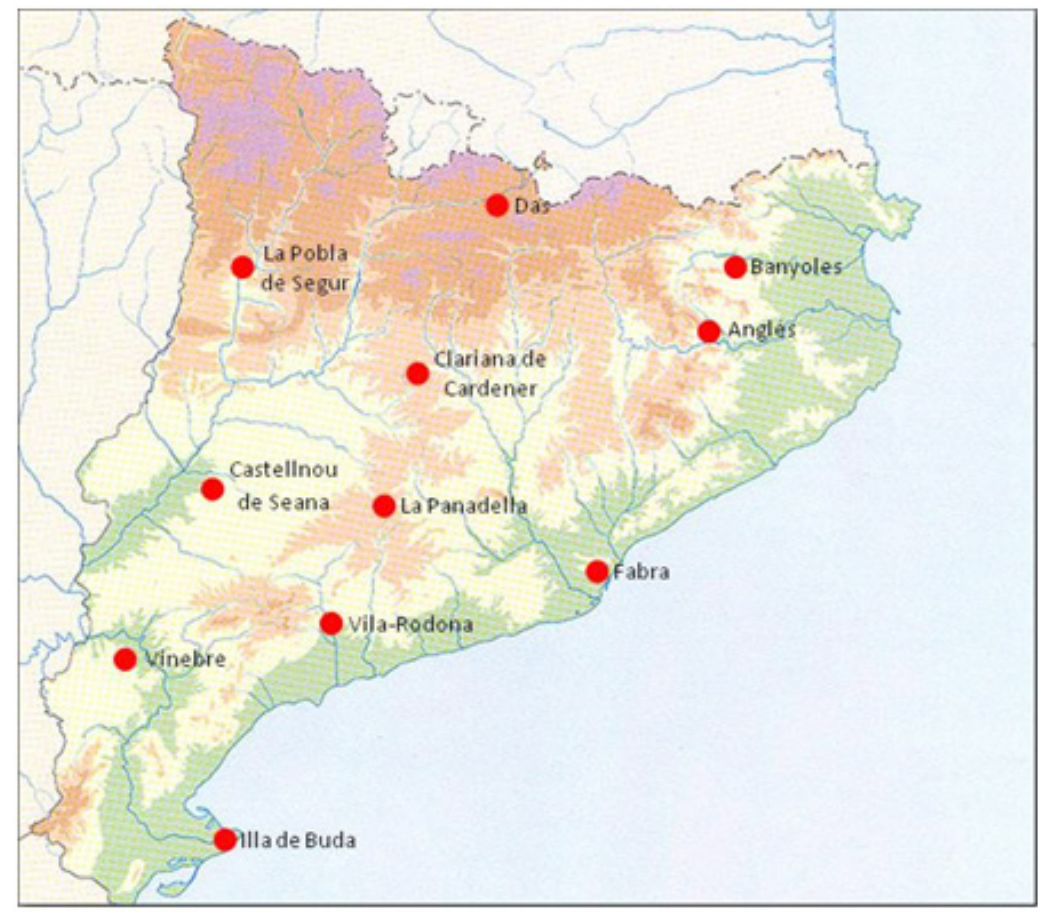

Fig. 1. Distribución de los observatorios utilizados.

Fig. 1. Distribution of observatories used. 
Tabla 1. VALORES DE LOS CONTEOS DE LOS DIFERENTES INTERVALOS CON PRECIPITACIÓN DURANTE 2010.

Table 1. Number OF PERIOdS With ReCORded PRECIPITATION DURING 2010.

\begin{tabular}{|c|c|c|c|c|c|c|c|c|c|c|c|}
\hline & Observatorio & $\begin{array}{c}30 \\
\min \end{array}$ & $\begin{array}{c}1 \\
\text { hora }\end{array}$ & \begin{tabular}{|c|}
2 \\
horas
\end{tabular} & \begin{tabular}{|c|}
3 \\
horas
\end{tabular} & \begin{tabular}{|c|} 
\\
horas
\end{tabular} & \begin{tabular}{|c|}
6 \\
horas \\
\end{tabular} & \begin{tabular}{|c|}
8 \\
horas
\end{tabular} & $\begin{array}{c}12 \\
\text { horas }\end{array}$ & \begin{tabular}{|c|}
24 \\
horas
\end{tabular} & $\begin{array}{c}48 \\
\text { horas }\end{array}$ \\
\hline Año completo & \multirow{3}{*}{ Anglès } & 1046 & 646 & 414 & 335 & 290 & 227 & 199 & 161 & 115 & 89 \\
\hline $\begin{array}{c}\text { Semestre } \\
\text { cálido }\end{array}$ & & 475 & 305 & 201 & 168 & 146 & 114 & 103 & 82 & 56 & 44 \\
\hline Semestre frío & & 571 & 341 & 213 & 167 & 144 & 113 & 96 & 79 & 59 & 45 \\
\hline Año completo & \multirow{3}{*}{ Banyoles } & 998 & 620 & 405 & 312 & 269 & 224 & 191 & 154 & 118 & 83 \\
\hline $\begin{array}{l}\text { Semestre } \\
\text { cálido }\end{array}$ & & 451 & 293 & 197 & 153 & 133 & 112 & 95 & 76 & 57 & 39 \\
\hline Semestre frío & & 547 & 327 & 208 & 159 & 136 & 112 & 96 & 78 & 61 & 44 \\
\hline Año completo & \multirow{3}{*}{$\begin{array}{l}\text { Castellnou } \\
\text { de Seana }\end{array}$} & 678 & 432 & 276 & 230 & 190 & 173 & 145 & 129 & 103 & 77 \\
\hline $\begin{array}{l}\text { Semestre } \\
\text { cálido }\end{array}$ & & 237 & 161 & 105 & 90 & 72 & 70 & 65 & 51 & 42 & 33 \\
\hline Semestre frío & & 441 & 271 & 171 & 140 & 118 & 103 & 80 & 78 & 61 & 44 \\
\hline Año completo & \multirow{3}{*}{$\begin{array}{l}\text { Clariana de } \\
\text { Cardener }\end{array}$} & 880 & 559 & 374 & 299 & 257 & 210 & 182 & 151 & 118 & 85 \\
\hline $\begin{array}{l}\text { Semestre } \\
\text { cálido }\end{array}$ & & 350 & 229 & 161 & 133 & 116 & 98 & 87 & 72 & 59 & 42 \\
\hline Semestre frío & & 530 & 330 & 213 & 166 & 141 & 112 & 95 & 79 & 59 & 43 \\
\hline Año completo & \multirow{3}{*}{ Das } & 899 & 586 & 398 & 326 & 75 & 37 & 215 & 170 & 127 & 92 \\
\hline $\begin{array}{l}\text { Semestre } \\
\text { cálido }\end{array}$ & & 488 & 320 & 217 & 179 & 153 & 129 & 118 & 90 & 69 & 48 \\
\hline Semestre frío & & 411 & 266 & 181 & 147 & 122 & 108 & 97 & 80 & 58 & 44 \\
\hline Año completo & \multirow{3}{*}{ Fabra } & 1016 & 635 & 415 & 325 & 273 & 215 & 183 & 154 & 117 & 80 \\
\hline $\begin{array}{c}\text { Semestre } \\
\text { cálido }\end{array}$ & & 363 & 233 & 160 & 131 & 114 & 93 & 83 & 71 & 56 & 39 \\
\hline Semestre frío & & 653 & 402 & 5 & 34 & 59 & 22 & bo & 83 & 61 & 41 \\
\hline Año completo & \multirow{3}{*}{ Illa de Buda } & 705 & 456 & 306 & 248 & 222 & 91 & 67 & 147 & 117 & 84 \\
\hline $\begin{array}{l}\text { Semestre } \\
\text { cálido }\end{array}$ & & 234 & 164 & 112 & 99 & 87 & 77 & 65 & 60 & 48 & 34 \\
\hline Semestre frío & & 471 & 292 & 194 & 149 & 135 & 114 & 102 & 87 & 69 & 50 \\
\hline Año completo & \multirow{3}{*}{ La Panadella } & 836 & 527 & 342 & 275 & 232 & 206 & 171 & 151 & 116 & 85 \\
\hline $\begin{array}{l}\text { Semestre } \\
\text { cálido }\end{array}$ & & 296 & 196 & 132 & 112 & 93 & 88 & 72 & 62 & 50 & 34 \\
\hline Semestre frío & & 540 & 30 & 2 & 163 & 39 & 8 & 99 & 05 & 66 & 1 \\
\hline Año completo & \multirow{3}{*}{$\begin{array}{l}\text { La Pobla de } \\
\text { Segur }\end{array}$} & 900 & 596 & 395 & 321 & 274 & 221 & 198 & 166 & 114 & 84 \\
\hline $\begin{array}{c}\text { Semestre } \\
\text { cálido }\end{array}$ & & 362 & 243 & 168 & 139 & 124 & 98 & 93 & 77 & 55 & 41 \\
\hline Semestre frío & & 538 & 353 & 227 & 182 & 150 & 123 & 105 & 89 & 59 & 43 \\
\hline Año completo & \multirow{3}{*}{ Vila-Rodona } & 752 & 488 & 325 & 264 & 228 & 188 & 158 & 138 & 102 & 76 \\
\hline $\begin{array}{l}\text { Semestre } \\
\text { cálido }\end{array}$ & & 257 & 178 & 124 & 103 & 87 & 75 & 66 & 58 & 44 & 31 \\
\hline Semestre frío & & 495 & 310 & 201 & 161 & 141 & 113 & 92 & 80 & 58 & 45 \\
\hline Año completo & \multirow{3}{*}{ Vinebre } & 629 & 396 & 264 & 215 & 191 & 162 & 147 & 123 & 99 & 74 \\
\hline $\begin{array}{l}\text { Semestre } \\
\text { cálido }\end{array}$ & & 219 & 143 & 96 & 79 & 73 & 61 & 58 & 47 & 39 & 31 \\
\hline Semestre frío & & 410 & 253 & 168 & 136 & 118 & 101 & 89 & 76 & 60 & 43 \\
\hline
\end{tabular}




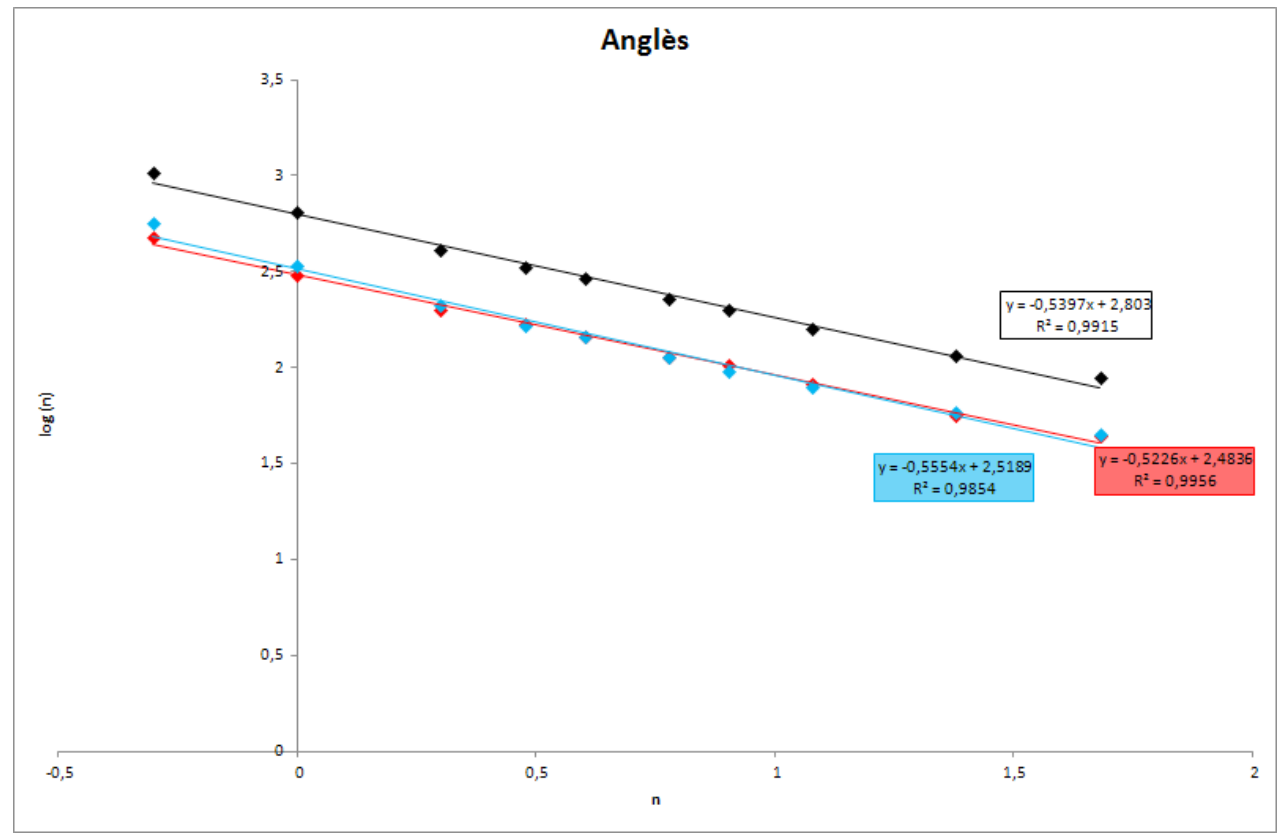

Fig. 2. Ajustes gráficos de los logaritmos de n y de l(n) para el caso de Anglès durante 2010 (en negro), durante el semestre cálido (en rojo) y durante el semestre frío (en azul).

Fig. 2. Graphics settings logarithms of $n$ and $I$ (n) for the case of Angles in 2010 (in black) during the warm semester (in red) and during the cold season (in blue).

Tabla 2. Valores de D y de $\mathbf{R}^{2}$ PARa los observatorios estudiados.

TABle 2. D ANd $\mathbf{R}^{2}$ VAlues FOR THE SELECTED OBSERVATORIES.

\begin{tabular}{|c|c|c|c|c|c|c|c|c|c|c|c|c|}
\hline & & $\frac{\sqrt[0]{0}}{\frac{0}{5}}$ & 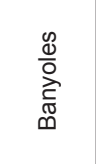 & 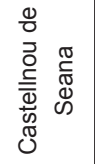 & 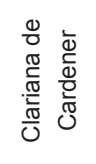 & $\begin{array}{l}\mathscr{0} \\
\tilde{\pi}\end{array}$ & $\begin{array}{l}\frac{\pi}{0} \\
\frac{\pi}{\pi} \\
\stackrel{\leftarrow}{\leftarrow}\end{array}$ & $\begin{array}{l}\frac{\pi}{0} \\
0 \\
0 \\
\frac{0}{0} \\
\underline{0}\end{array}$ & $\begin{array}{l}\frac{\pi}{0} \\
\overline{0} \\
\frac{\pi}{0} \\
\frac{\pi}{\pi} \\
0 \\
\widetilde{J}\end{array}$ & 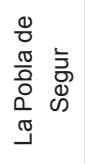 & 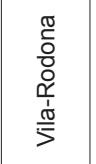 & 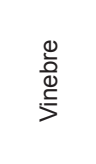 \\
\hline \multirow{2}{*}{$\begin{array}{c}\text { Año } \\
\text { completo }\end{array}$} & $\begin{array}{c}\text { Valor de } \\
\mathrm{R}^{2}\end{array}$ & 0,9915 & 0,9913 & 0,9763 & 0,9906 & 0,9947 & 0,9919 & 0,984 & 0,9837 & 0,9966 & 0,9922 & 0,9813 \\
\hline & $\begin{array}{c}\text { Dimensión } \\
\text { fractal }\end{array}$ & 1,5397 & 1,5362 & 1,4632 & 1,5048 & 1,4893 & 1,5504 & 1,4479 & 1,4883 & 1,5159 & 1,4977 & 1,4531 \\
\hline \multirow{2}{*}{$\begin{array}{c}\text { Semestre } \\
\text { cálido }\end{array}$} & $\begin{array}{c}\text { Valor de } \\
\mathrm{R}^{2}\end{array}$ & 0,9854 & 0,9965 & 0,9753 & 0,9913 & 0,9961 & 0,9906 & 0,9894 & 0,9889 & 0,9959 & 0,9947 & 0,9762 \\
\hline & $\begin{array}{c}\text { Dimensión } \\
\text { fractal }\end{array}$ & 1,5554 & 1,5279 & 1,4223 & 1,4512 & 1,4966 & 1,4735 & 1,4052 & 1,4554 & 1,4693 & 1,4523 & 1,417 \\
\hline \multirow{2}{*}{$\begin{array}{c}\text { Semestre } \\
\text { frío }\end{array}$} & $\begin{array}{c}\text { Valor de } \\
R^{2}\end{array}$ & 0,9956 & 0,9843 & 0,9732 & 0,9905 & 0,9917 & 0,993 & 0,9798 & 0,9781 & 0,9967 & 0,9895 & 0,9836 \\
\hline & $\begin{array}{c}\text { Dimensión } \\
\text { fractal }\end{array}$ & 1,5226 & 1,5435 & 1,4896 & 1,5487 & 1,4808 & 1,6066 & 1,4735 & 1,51 & 1,5533 & 1,5263 & 1,4754 \\
\hline \multicolumn{2}{|c|}{$\begin{array}{c}\text { Diferencia de los } \\
\text { valores de } D \text { de los } \\
\text { semestres cálido y frío }\end{array}$} & 0,0328 & $-0,0156$ & $-0,0673$ & $-0,0975$ & 0,0158 & $-0,1331$ & $-0,0683$ & $-0,0546$ & $-0,084$ & $-0,074$ & $-0,0584$ \\
\hline
\end{tabular}


Comportamiento espacial de la dimensión fractal

Al considerar los once observatorios repartidos homogéneamente por el área de estudio, es posible obtener una representación del comportamiento espacial de la variable D sobre el territorio, ya sea a nivel anual (Fig. 3), en su semestre cálido (Fig. 4) y en el frío (Fig. 5), o de la diferencia entre ambos (Fig. 6).

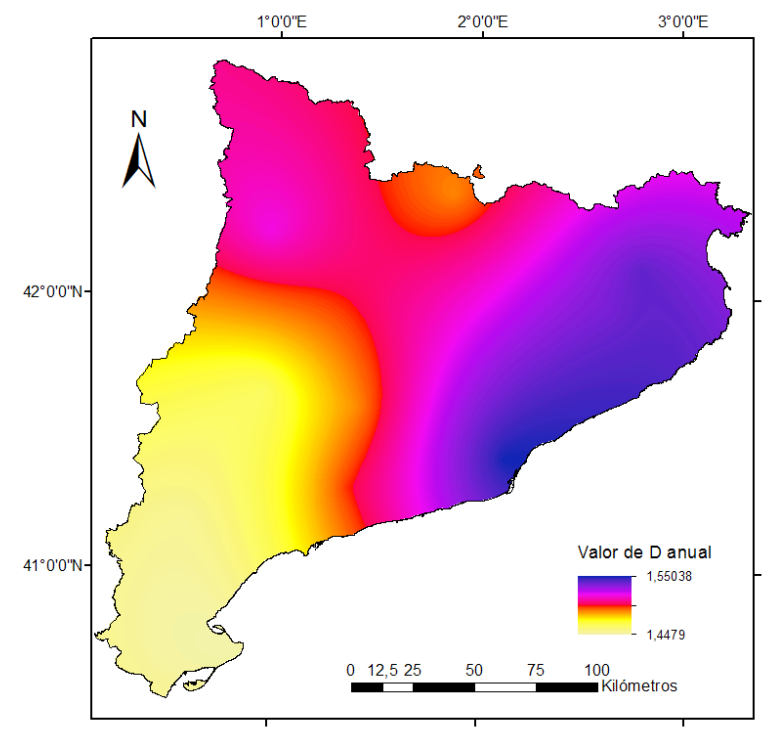

Fig. 3. Valores de D (dimensión fractal) anual en Cataluña en 2010.

Fig. 3. Values of D Annual (fractal dimension) in Catalonia in 2010.

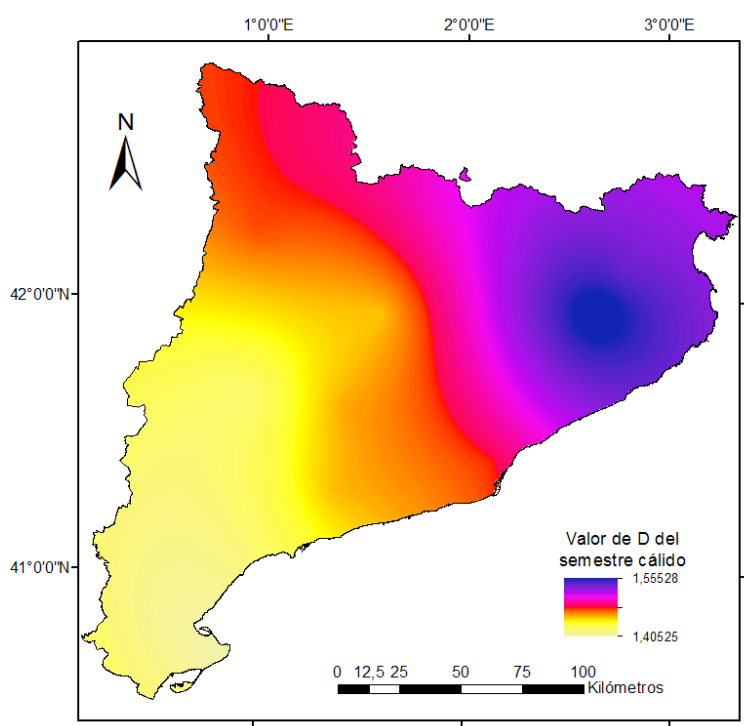

Fig. 4. Valores de D (dimensión fractal) del semestre cálido en Cataluña en 2010.

Fig. 4. Values of D (fractal dimension) of the warm semester in Catalonia in 2010. 


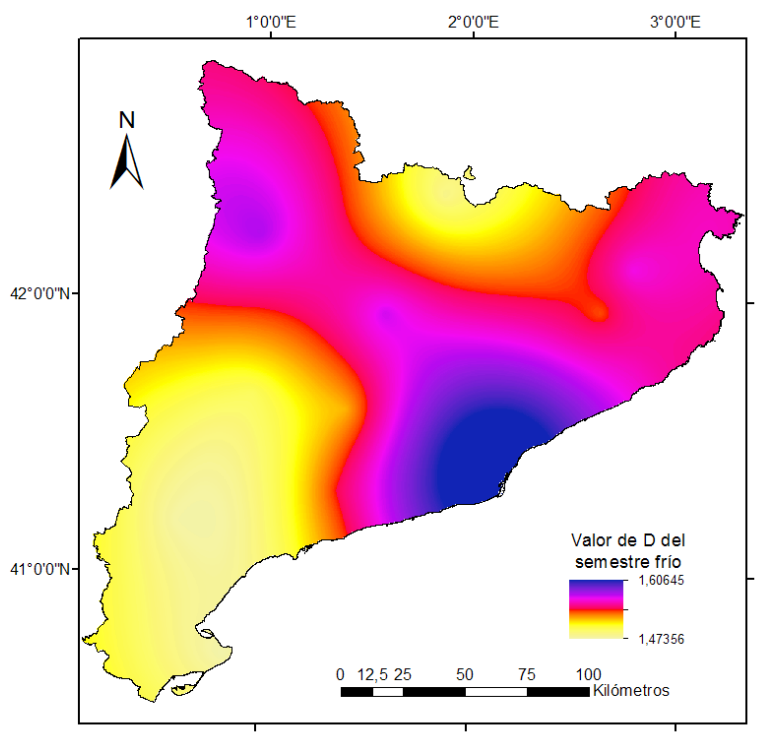

Fig. 5. Valores de D (dimensión fractal) del semestre frío en Cataluña en 2010.

Fig. 5. Values of D (fractal dimension) of the cold season in Catalonia in 2010.

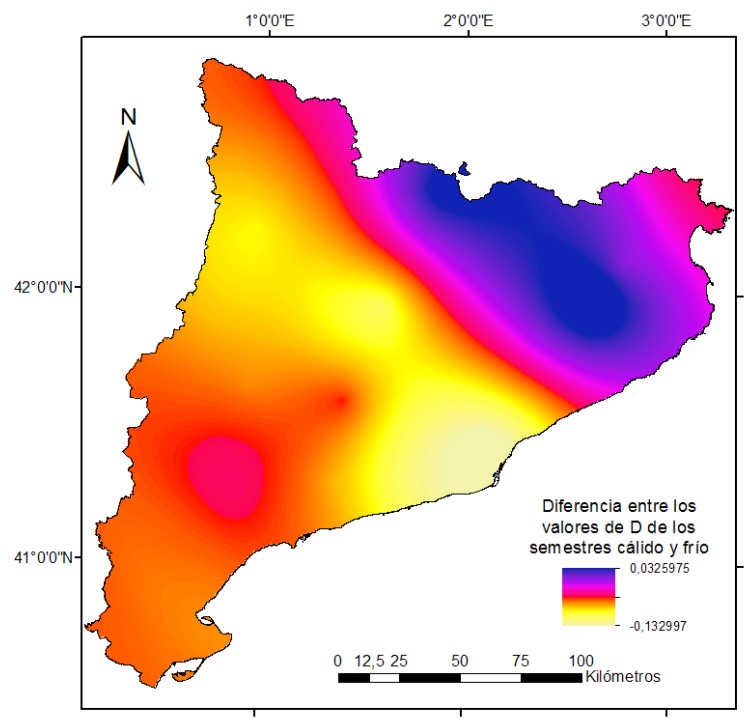

Fig. 6. Valores de las diferencias entre D del semestre cálido menos D del semestre frío en Cataluña en 2010.

Fig. 6. Values of the differences between $D$ the warm half cold half less D in Catalonia in 2010

Los valores mínimos de D anualmente se concentran en la parte más meridional del territorio, en su vértice suroriental, aumentando paulatinamente con la latitud y con una aparente influencia marítima, siendo mayores cerca de la costa si consideramos la mitad norte de la región. El mismo patrón, con aumento a lo largo de 
un eje de suroeste a nordeste, se repite en el caso del semestre cálido, no así durante el semestre frío, donde no parece que haya una influencia latitudinal, registrándose los valores más altos en la costa central, y los valores más bajos en el sur y en el norte. En cuanto a la diferencia entre los semestres cálido y frío, no existe un patrón claro que explique los resultados obtenidos, pero sí parece que los escasos observatorios con una $\mathrm{D}$ en el semestre cálido superior a la del semestre frío, o próxima, se concentran en el nordeste de la región.

\section{DISCUSIÓN}

Es posible llevar el estudio de la dimensión fractal de la distribución temporal de la precipitación a nuevos enfoques gracias a la instalación y el desarrollo de las redes de observatorios meteorológicos automáticos, que son capaces de registrar con precisión datos con una resolución temporal fina, que no permiten las estaciones manuales convencionales. Este hecho permite llevar a cabo nuevas aproximaciones al concepto de distribución fractal de la precipitación, así como el cálculo de las dimensiones fractales con una mayor base empírica. Fenómenos como las precipitaciones de carácter convectivo, que a menudo tienen duraciones sub-horarias, la persistencia horaria de la lluvia y hasta sus tendencias pueden ser abordables estadísticamente a través del concepto de fractalidad temporal.

La determinación de los valores de las dimensiones fractales para los once observatorios de Cataluña en el año 2010, constituye un primer ejercicio que se seguirá con el mismo análisis para períodos más largos. El hecho de no existir apenas estudios sobre el comportamiento fractal temporal de la precipitación sobre datos a resolución temporal fina (sub-horaria), hace difícil la comparación de estos resultados con otros. Sin embargo, sí es posible realizar analogías del comportamiento de la precipitación a diferentes escalas temporales.
La localización del área de estudio, en latitudes templadas-subtropicales y de cara a un gran mar interior como es el Mediterráneo, provoca que los procesos que están en el origen de la precipitación varíen dependiendo de la época del año. Así, en los meses fríos, la precipitación va asociada al paso de frentes polares provenientes del oeste, dando lugar a una precipitación regular y de escasa intensidad horaria. Por el contrario, en la mitad cálida del año la precipitación tiene un origen predominantemente convectivo, con episodios muy concentrados en el tiempo. Estos procesos explican las diferencias de los valores de las dimensiones fractales entre el semestre cálido y el semestre frío, mayores en este último.

Que los mayores valores de la dimensión fractal a nivel anual se den a mayor latitud podría estar relacionado con la mayor exposición de este sector a la circulación del oeste que el sector más meridional, donde se registra un valor de la dimensión fractal más bajo. En consonancia con lo hallado por PÉREZ et al. (2011), en el año 2010 en Cataluña las precipitaciones en invierno se han producido por un paso de frentes relativamente continuo, lo que probablemente ha mantenido la propiedad de auto-similitud de los fractales. Al tratarse únicamente del análisis de un año concreto, es posible que los patrones espaciales hayan quedado ocultos. Durante el semestre cálido los valores más bajos de $\mathrm{D}$, respecto al semestre frío, pueden revelar el carácter más convectivo de la precipitación, especialmente en el sur de la región, con los valores más bajos.

Sin embargo, el hecho de que en dos observatorios (Anglès y Das) los valores de la dimensión fractal sean mayores en los meses cálidos que en los meses fríos hace pensar que otros mecanismos, aparte de los puramente dinámicos, puedan intervenir en la regularidad y persistencia de la precipitación. Quizá la explicación habría que buscarla en motivos locales, en 
los que se den, por lo menos en el caso de Das, ascensos forzados por la orografía que otorguen mayor persistencia y repetición a la precipitación en verano.

\section{CONCLUSIONES}

La dimensión fractal de la distribución temporal de la precipitación, considerada como un índice de regularidad/irregularidad temporal de la precipitación, permite abordar un estudio de la lluvia a diferentes escalas temporales. En el estudio de caso, con datos semi-horarios, la dimensión fractal aporta una visión distinta del comportamiento temporal fino de la precipitación durante el año 2010, relacionada por definición con la auto similitud estadística respecto al tiempo.

Las dimensiones fractales de los 11 observatorios de Cataluña durante 2010 van de 1,4479 en el extremo meridional de la región a 1,5504 en la costa central (Barcelona). En el semestre cálido van de 1,4052 en el extremo sur a 1,5554 en el nordeste y en el semestre frío, de 1,4735 a 1,6066 entre los mismos lugares citados para el caso anual. Los valores son mayores en el semestre frío que en el cálido, excepto en dos observatorios. La pauta espacial más clara es la de un aumento de los valores de D desde el suroeste al nordeste en el conjunto del año y en verano.

Como trabajo de futuro, además de aumentar el período de análisis a varios años, se recurrirá a la teoría multifractal, dado que en la naturaleza existen determinados procesos geofísicos y sistemas que no pueden estudiarse como conjuntos simples, ya que se caracterizan por diferentes niveles de intensidad, y no pueden describirse simplemente en función de, por ejemplo, su ocurrencia o no ocurrencia. La teoría multifractal facilita el estudio de procesos no lineales que presentan diferentes niveles de intensidad. En lugar de trabajar con una escala simple (como en el caso de los fractales), se utiliza el concepto de escala múltiple. El término multifractal hace referencia a medidas caracterizadas por un conjunto infinito de índices de escala o dimensiones fractales (FRISCH \& PARISI, 1985). Desde esta perspectiva se quiere abordar el análisis de la fractalidad temporal de la precipitación en el futuro.

\section{AGRADECIMIENTOS}

Esta investigación está financiada por el Programa de Formación de Profesorado Universitario del Ministerio de Educación del Gobierno de España. Además, también se inserta dentro del proyecto PRECABAL (CGL2011-29263-C02-01) del Ministerio de Ciencia e Innovación del Gobierno de España, y en el marco de las investigaciones del Grupo de Climatología de la Universidad de Barcelona (2009 SGR 443) y de su Institut de l'Aigua.

\section{REFERENCIAS}

AMARO, I.R., J.R. DEMEY \& R. MACCHIAVELLI, 2004. Aplicación del Análisis R/S de Hurst para estudiar las propiedades fractales de la precipitación en Venezuela. Interciencia, 29: 617-620.

FRISCH, U. \& G. PARISI, 1985. Fully developed turbulence and intermittency, en FRISCH,U.\&PARISI, G.(Eds.), Turbulence and Predictability in Geophysical Fluid Dynamics. International School of Physics Enrico Fermi, Amsterdam: 359-367.

HSUI A.T., K.A. RUST \& G.D. KLEIN, 1993. A fractal analysis of Quaternary, Cenozoic-Mesozoic, and Late Pennsylvanian sea level changes. Journal of Geophysical Research, 98: 21963-21967. 
MANDELBROT, B.B., 1977. The Fractal Geometry of Nature. W.H. Freeman and Company, New York.

OÑATE RUBALCABA， J.J., 1997. Fractal Analysis of Climatic Data: Annual Precipitation Records in Spain. Theoretical and Applied Climatology, 56: 83-87.

PÉREZ, S.P., E.M. SIERRA, M.J. MASSOBRIO \& F.R. MOMO, 2009. Análisis fractal de la precipitación anual en el este de la Provincia de la Pampa, Argentina. Revista de Climatología, 9: 25-31.
SCHROEDER, K., J. GARCÍALAFUENTE, S.A. JOSEY, V. ARTALE, B. BUONGIORNO NARDELLI, A. CARRILLO, M. GAČIĆ, G.P. GASPARINI, M. HERRMAN, P. LIONELLO, W. LUDWIG, C. MILLOT, E. ÖZSOKY, G. PISACANE, J.C. SÁNCHEZ-GARRIDO, G. SANNINO, R. SANTOLERI, S. SOMOT, M. STRUGLIA, E. STANEV, I. TAUPIERLETAGE, M.N. TSIMPLIS, M. VARGAS-YÁÑEZ，V. ZERVAKIS \& G. ZODIATIS 2012. Circulation of the Mediterranean Sea and it's Variability, en PILONELLO, P. (Ed.): The Climate of the Mediterranean Region. From the Past to the Future, Elsevier Insights, London: 187-256.

\section{Imagen de Investigaciones GEOGRÁFICAS}

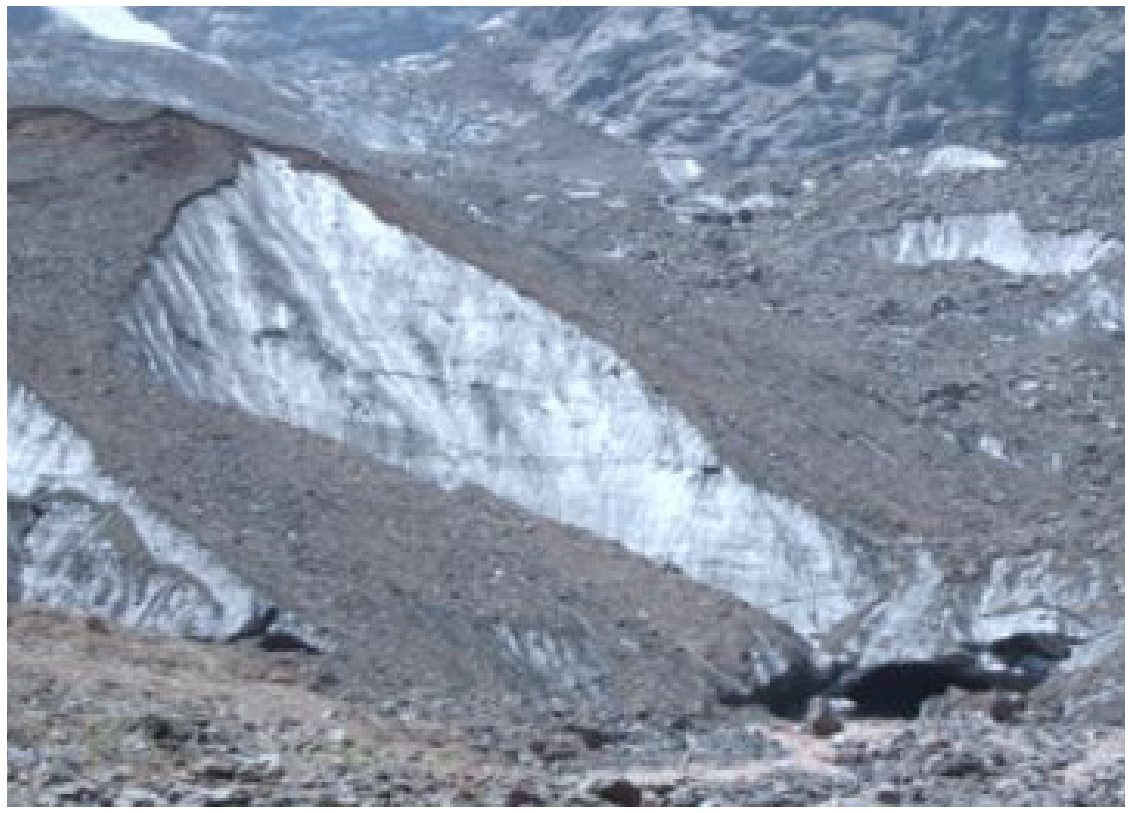

El Glaciar de El Morado, en el parque Andino del mismo nombre, es un claro ejemplo de los efectos que el calentamiento global está teniendo en los glaciares de alta montaña de latitudes medias, donde los registros térmicos indican $1^{\circ} \mathrm{C}$ de calentamiento en los últimos 40 años (Estación El Yeso). Ubicado en la cuenca superior del Río Maipo, Región Metropolitana, y a unos 2600 m.s.n.m, muestra una superficie quebrada producto por fuertes colapsos internos y una cubierta detrítica de varios decímetros de espesor que actúa como un efectivo trasmisor de la alta radiación solar sobre el cuerpo de hielo. Su frente presenta a lo menos tres puertas glaciarias, evidencia de los abundantes flujos hídricos que circulan por su interior, lo cual es otra evidencia del claro balance de masa negativo que presenta. Igual situación se registra en otros glaciares cubiertos de este sector andino, como es el caso del Glaciar Pirámide.

Dr. Prof. Ferrando Acuña, Departamento de Geografía, Universidad de Chile, fferrand@uchilefau.cl 